\title{
Gradient-based non-linear microstructure design
}

Brent L. Adams

b_I_adams@byu.edu

M. Lyon

Follow this and additional works at: https://scholarsarchive.byu.edu/facpub

Part of the Mechanical Engineering Commons

Original Publication Citation

Journal of the Mechanics and Physics of Solids 52 (24) 2569-2586

\section{BYU ScholarsArchive Citation}

Adams, Brent L. and Lyon, M., "Gradient-based non-linear microstructure design" (2004). Faculty Publications. 445.

https://scholarsarchive.byu.edu/facpub/445 


\title{
Gradient-based non-linear microstructure design
}

\author{
M. Lyon*, B.L. Adams \\ Department of Mechanical Engineering, Brigham Young University, 435 CTB, Provo, UT 84602, USA
}

Received 3 January 2003; received in revised form 2 February 2004; accepted 21 April 2004

\begin{abstract}
Fourier analysis is implemented on the orientation distribution of a polycrystalline microstructure. The linearity and convexity of the Fourier space, with respect to orientation, allows one to consider all possible distributions by considering all linear combinations of single-grain orientations. The limits of the Fourier space are therefore defined by the solutions to a set of linear programming problems. A unique approach to the linear programming, similar to the Krylov subspace methods for obtaining solutions to linear systems, is presented. The method is particularly efficient for this application where a large number of independent variables is often required. These solutions are then used as the constraints in the gradient-based optimization of non-linear functions within the Fourier space. In the example, Taylor yield theory and an anisotropic solution for the stress concentration around a hole in a plate of cubic-orthotropic polycrystalline material are expressed as non-linear functions within the Fourier space. The maximum obtainable ratio of Taylor factor to stress concentration for any polycrystalline orientation distribution in copper is found to be 1.22 , more than double the minimum value.
\end{abstract}

(C) 2004 Elsevier Ltd. All rights reserved.

Keywords: A. Polycrystalline material; B. Optimization; C. Anisotropic material; D. Microstructures; E. Metal plasticity

\section{Introduction}

In highly constrained design, the material is often the limiting factor. The designer is faced with few alternatives and must occasionally use expensive single-crystal components in applications where a material's anisotropy must be exploited. In an effort to give the designer access to the broader range of properties available from the anisotropy generated by less expensive processing routes, microstructure sensitive design (MSD)

\footnotetext{
* Corresponding author. Applied and Computational Mathematics, California Institute of Technology, MC 217-50, Pasadena 91125, USA. Tel.: +1-626-3954775; fax: +1-801-4220516.

E-mail addresses: mlyon@byu.edu (M. Lyon), b_1_
} 
was developed (Adams et al., 2001, 2004). The framework of MSD allows the designer to see what properties are available from all possible microstructures, as defined by the distributions of their local states in accordance with selected homogenization theory. The designer along with a materials processing specialist can then choose a feasible local state distribution that is ideal for the application. Eventually, known processing paths could be optimized in order to obtain previously infeasible microstructures (Kalidindi et al., 2002).

The general theory behind linear MSD incorporates complex local states including, but not limited to: composition, phase, lattice orientation and elements of distributions pertaining to higher order homogenization theory (Adams et al., 2004). A Fourier or spectral series representation of the local state space is developed according to orthonormal basis functions on the special orthogonal group $S O(3)$ and scalar spaces (Bunge, 1965; Roe, 1965; Keener, 2000). A convex region in that space was identified as the microstructure hull. This hull is defined to be the set of all points representing Fourier coefficients of all possible local state distributions. Certain material properties could then be expressed as hyperplanes passing through the microstructure hull. The subspace created by the intersection of the hyperplanes with the microstructure hull is a convex subspace, within which a linear programming problem can be formulated to find the optimum of properties and coefficients of the local state distribution that would realize those properties. This was the focus of the original work in MSD (Adams et al., 2001, 2004).

By the definition of the microstructure hull, a necessary and sufficient condition for the existence of a particular local state distribution with optimal properties is that the coefficients of the Fourier expansion of the distribution lie within the hull. The microstructure hull then provides a continuous space which can be explored to find distributions with optimal properties for a given design problem. In other words MSD can be used to determine a microstructure that will exhibit properties that are ideal for a specific design application. This gives the designer full freedom to exploit a materials microstructure to improve design.

This paper extends MSD to allow for properties that have a non-linear functional dependence on the coefficients of the series. Intersections between these properties and the microstructure hull no longer provides a convex subspace and therefore the approach taken by Adams et al. (2004) can no longer be used. Solutions to these problems are formulated in this paper by redefining the linear programming problem to determine the limits of the microstructure hull. Within the microstructure hull, a variety of non-linear solvers can be used to efficiently locate microstructures of interest. The point to which the non-linear solver converges is guaranteed to represent a real microstructure if the optimization is forced to remain inside the microstructure hull. A main focus of this paper is an algorithm that determines the limits of the convex microstructure hull in a given direction. This algorithm, which has some similarities to the Krylov subspace methods for the solving of linear systems, runs extremely efficiently. Using the limits determined by this algorithm, the non-linear solver is restricted to find only coefficients for feasible local state distributions.

To illustrate the method, it will be applied to a thin orthotropic copper plate with a circular hole with a diameter much smaller than the width of the plate. This paper 
focuses on the one-point probability distribution of crystallite orientation, although the method can be applied to the general theory of MSD with more complex local states and more complex descriptions of the spatial correlations of the distribution. The plate is axially loaded in a principal direction of its orthotropy and stress concentrations develop around the hole. The magnitude of the stress concentration was found by Leknitskii (1963) to be dependent on the anisotropic elastic properties of the material. Taylor yield theory is added to the model to obtain a prediction of the initial yield.

The problem of finding the optimal microstructure for the given conditions has been completed by Henrie et al. (2002) and Kalidindi et al. (2002). The former used a simulated annealing algorithm to explore all possible microstructures and find optimal solutions. The latter used a guided search to obtain approximate results. In neither case were the linear properties of the Fourier space and microstructure hull fully exploited. The current method is more efficient and able to obtain more accurate solutions. The results of this method were compared to both of the preceding solutions, and good agreement was obtained with the former. It should be noted that the current method is easily adapted to more complex problems of determining optimal distributions of continuous functions in material science and in general.

This works considers any possible local state distribution feasible and attempts to find an optimal distribution for specific design requirements. But of course not all possible distributions are obtainable. The purpose of this work is then to motivate the development of new processing methods by determining desirable microstructures, and to show the improvement in design than can be achieved by fully exploiting the microstructure. Future work will constrain the optimization direction to lie directly on known processing paths. Each step in the optimization will then correspond to a particle process being used to prepare the material. By limiting the number of steps in the process, control of production cost of the material could be maintained. By constraining MSD in this way, only obtainable local state distributions would be calculated.

\section{The microstructure hull as a constrained Fourier space}

This paper focuses on single-phase cubic polycrystals possessing orthorhombic statistical symmetry. The assumption of orthorhombic statistical symmetry is required in order to apply Leknitskii's solution for the stress concentration factor but it is anticipated that part of the processing required to obtain a thin plate would involve a rolling process which would justify this assumption. The assumption of cubic crystal symmetry reduces the dimensionality of the microstructure hull making the computations faster. The framework of MSD does not require any symmetry to be present and can be treated in general. But whenever symmetries are present, the method can be formulated to reduce computation by exploiting those symmetries. A function that is linearly dependent upon the grain orientation can be expressed as a Fourier series of generalized spherical harmonic functions $T_{l}^{m n}(g)$, which provide a complete orthonormal basis on $S O(3)$. These functions have been subsequently symmetrized according to cubic crystal symmetry and orthorhombic sample symmetry so that they constitute an orthonormal basis over the homogenous space $S O(3) / G$, where $G$ is the symmetry 
subgroup of the crystal lattice (Bunge, 1993; Brocker and tom-Dieck, 1985). Using the cubic-orthorhombic symmetric version of the generalized spherical harmonics (following the notation of Bunge, 1993, this particular symmetry case is denoted by three dots above the function), the orientation distribution function $f(g)$ can be expressed as,

$$
f(g)=\sum_{l=0}^{\infty} \sum_{m=1}^{M(l)} \sum_{n=1}^{N(l)} \dot{T}_{l}^{m n}(g) F_{l}^{m n}
$$

where $f(g) \mathrm{d} g$ is the volume fraction of material with orientation $g$ lying within the range $\mathrm{d} g$ of $g$. The limits of summation in relation (1), $M(l)$ and $N(l)$, are obtained by consideration of the elements of cubic-orthorhombic symmetry, and have been described in detail by Bunge (1993). The Fourier coefficients, $F_{l}^{m n}$, can be determined by multiply each side by the complex conjugate of a particular harmonic function denoted $\dot{\bar{T}}_{l^{\prime}}^{* m^{\prime} n^{\prime}}(g)$ and then integrating,

$$
\iiint_{S O(3) / G} f(g) \dot{\vec{T}}_{l^{\prime}}^{* m^{\prime} n^{\prime}}(g) \mathrm{d} g=\iiint_{S O(3) / G} \sum_{l=0}^{\infty} \sum_{m=1}^{M(l)} \sum_{n=1}^{N(l)} \dot{\vec{T}}_{l}^{m n}(g) \dot{\vec{T}}_{l^{\prime}}^{* m^{\prime} n^{\prime}}(g) F_{l}^{m n} \mathrm{~d} g .
$$

For a distribution approaching that of a single orientation, $\delta\left(g_{i}\right)$, the integral on the left vanishes except for a single term and due to the orthonormality of generalized spherical harmonic functions, and Eq. (2) reduces to

$$
F_{l}^{m n}=(2 l+1) \dot{\bar{T}}_{l}^{* m n}\left(g_{i}\right) .
$$

Using a vector analogy, the coefficients $F_{l}^{m n}$ are the components of the vector locating the orientation $g_{i}$ within a Fourier space of dimension defined by the truncation of the series with the cubic symmetric generalized spherical harmonic functions as the basis vectors of the space.

For a bi-crystal distribution, the orientation is given by

$$
f(g)=v_{A} \delta\left(g_{A}\right)+v_{B} \delta\left(g_{B}\right),
$$

with $v_{A}$ and $v_{B}$ as the volume fractions of material with orientation $g_{A}$ and $g_{B}$ respectively. Introducing this distribution into Eq. (2) yields

$$
F_{l}^{m n}=(2 l+1)\left[v_{A} \dot{\bar{T}}_{l}^{* m n}\left(g_{A}\right)+v_{B} \dot{\bar{T}}_{l}^{* m n}\left(g_{B}\right)\right] .
$$

In general, the $F_{l}^{m n}$ coefficients for any polycrystalline orientation distribution, $f(g)$, are obtained by linear combinations of the single-crystal coefficients or the location of single-crystal distributions within the Fourier space

$$
F_{l}^{m n}=(2 l+1) \sum_{i} v_{i} \dot{\bar{T}}_{l}^{* m n}\left(g_{i}\right)
$$

The above equation establishes the linearity of the Fourier space. The range of the coefficients is limited by the requirements that $g \in S O(3) / G, v_{i} \geqslant 0$, and $\sum_{i} v_{i}=1$. Consideration of all possible orientation distributions yields a convex volume in a multi-dimensional space representing all possible $F_{l}^{m n}$ coefficients for real distributions. 


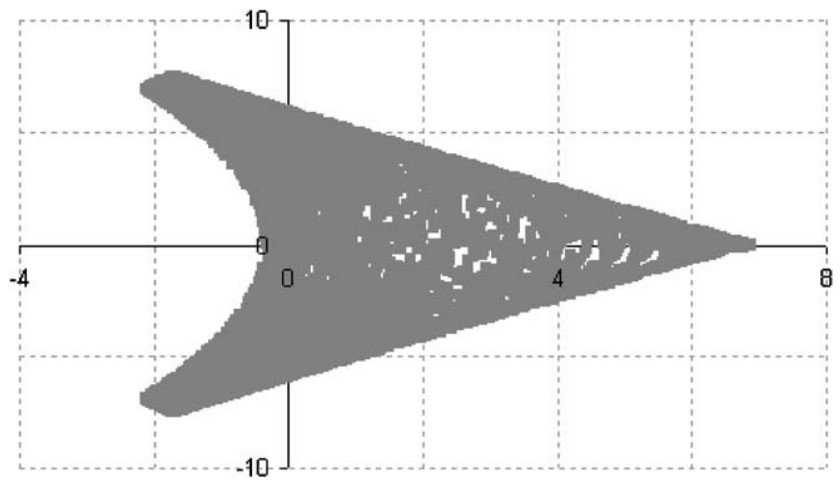

Fig. 1. Two-dimensional slice of the Fourier space showing the location of orientations in the fundamental zone according to Eq. (2). The slice is taken through the plane $F_{4}^{13}=4$. The $F_{4}^{11}$ and $F_{4}^{12}$ dimensions are shown. Label the axes on the figure!

Since the series expansion of the orientation distribution function, Eq. (1), goes to infinity, the series must be truncated in practical applications to some finite dimension. With truncation, the coefficients cease to represent single distinguishable orientation distributions. Instead, a family of orientation distributions can be found for each set of $F_{l}^{m n}$ coefficients, which have the same location within the selected finite dimensional subspace of the full Fourier space.

A two-dimensional slice through the Fourier space is shown in Fig. 1. The fundamental zone of orientations, $S O(3) / G$, was discretized into several thousand orientations. For each of these orientations the coefficients $F_{l}^{m n}$ were calculated by Eq. (3). Due to the cubic-orthorhombic symmetry of the basis functions, the first three non-zero coefficients are $F_{4}^{11}, F_{4}^{12}$, and $F_{4}^{13}$. For each orientation with $F_{4}^{13} \cong 4$, the values of $F_{4}^{11}$ and $F_{4}^{12}$ were plotted in Fig. 1 .

Due to the coarseness of the discretization of the possible orientations, Fig. 1 contains some small areas where nothing was plotted but would have been had the discretization been finer. In this subspace then, the coefficients of single orientations forms a triangle with two truncated corners and an approximately semicircular region missing. Since this region is determined from Eq. (3), it contains only single orientations.

Since polycrystalline distributions are linear combinations of the single-crystal distributions (Compare Eqs. (3) and (6)), the microstructure hull is actually larger than the region in Fig. 1. In fact a uniquely polycrystalline region exists within the Fourier space. The uniquely polycrystalline region is the lighter shade, and the darker shade is the region accessible by both the single-crystal and polycrystalline distributions. The union of the two regions is the microstructure hull. Historically, material solutions to highly constrained design problems have only exploited the properties of the darker region. By considering the entire microstructure hull, MSD is able to take advantage of the additional properties obtained only by polycrystalline orientation distributions without losing the properties of single crystals. 


\section{Gradient-based optimization within the Fourier space}

In order to illustrate the method for optimization of non-linear functions, a simple gradient search method is used. It will be apparent to those experienced in optimization that applying higher order algorithms, like the generalized reduced gradient and sequential quadratic programming methods, follow from this illustration. Other methods including simulated annealing and genetic algorithms could also be applied as long as they are held to the same constraints as the gradient methods.

The gradient of a given property $H(g)$ in the Fourier space of Eq. (1) takes the form,

$$
\nabla H(g)=\frac{\partial H(g)}{\partial F_{4}^{11}} \hat{F}_{4}^{11}+\frac{\partial H(g)}{\partial F_{4}^{12}} \hat{F}_{4}^{12}+\frac{\partial H(g)}{\partial F_{4}^{13}} \hat{F}_{4}^{13}+\frac{\partial H(g)}{\partial F_{6}^{11}} \hat{F}_{6}^{11}+\cdots,
$$

revealing the direction in which the function will increase the most in the Fourier space.

The algorithm that is used to optimize this distribution for the function has only a few basic steps. First, a feasible starting point is selected. Convenient choices include the location of any individual orientation as defined by Eq. (2), or the location of a completely random distribution for which all Fourier coefficients except the constant term are zero. From that location, a small step is taken in the direction of the gradient. The feasibility of this new point is analyzed by determining whether or not it can be expressed as a linear combination of a discrete set of individual orientations spanning the fundamental zone, $S O(3) / G$. This cycle is repeated until the objective function can no longer increase within the hull or the gradient is zero, denoting a maximum value of the function.

Implementing this algorithm therefore requires a method that determines whether or not a given location within the Fourier space is within the microstructure hull (i.e. can be expressed as a linear combination of the discrete set of individual orientations spanning $S O(3) / G)$. Due to the linear properties of the space as established in Eq. (6), linear programming methods can be applied to determine whether or not a given point is feasible. The linear programming algorithm used in this paper will be described in the next section.

If the gradient of the function passes out of the microstructure hull, the optimal within the hull has not necessarily been reached. Gram-Schmidt Orthonormalization is used to allow the optimization path to follow the boundary of the hull until the optimal solution is found. In Gram-Schmidt Orthonormalization, given two vectors, $\vec{v}_{1}$ and $\vec{v}_{2}$, an orthonormal basis can be constructed using the vectors, as long as they are linearly independent. The first basis vector can be defined as

$$
\hat{e}_{1}=\frac{\vec{v}_{1}}{\left\|\vec{v}_{1}\right\|} .
$$

The second basis is formed from the part of vector $\vec{v}_{2}$ that is orthogonal to $\vec{v}_{1}$. This is found by subtracting from $\vec{v}_{2}$ the projection of $\vec{v}_{2}$ on $\vec{v}_{1}$. The second basis vector is then defined by

$$
\hat{e}_{2}=\frac{\vec{v}_{2}-\left(\hat{e}_{1} \cdot \vec{v}_{2}\right) \hat{e}_{1}}{\left\|\vec{v}_{2}-\left(\hat{e}_{1} \cdot \vec{v}_{2}\right) \hat{e}_{1}\right\|} .
$$


If $\vec{v}_{1}$ and $\vec{v}_{2}$ were not independent, the denominator of Eq. (9) would be zero and the second basis vector would be undefined. This process can be continued into higher dimensional spaces.

Applied to the problem at hand, if $\vec{v}_{1}$ is taken to be the gradient direction and $\vec{v}_{2}$ to be the normal of the hyper-plane tangent to the microstructure hull at the exterior point of interest, then the direction $\hat{e}_{2}$ is the direction on the bounding hyper-plane in which the objective is increasing the most. If the denominator in Eq. (9) goes to zero and $\hat{e}_{2}$ becomes undefined, then there is no direction on the boundary of the hull that increases the objective and an optimal has been reached. This condition, along with the condition that the gradient goes to zero if an optimal point is reached within the hull, would be replaced by the Kuhn-Tucker conditions in a more sophisticated optimization approach (Rardin, 1998).

Naturally, this algorithm will suffer from all the drawbacks of gradient-based optimization, including trapping at local minima and maxima. But these can be handled by starting at a variety of initial points or by implementing non-linear optimization codes based on either simulated annealing or genetic algorithms, which are designed to handle these difficulties. Using a variety of initial points can be efficient since feasible initial points are readily obtained from Eq. (6). The actual methods used would need to be based on the specific property models and how many minima and maxima they produce in the Fourier Space.

\section{Bounding the microstructure hull with linear programming}

Linear programming is used to determine if a point in the space is within the microstructure hull. This is done by determining if the point can be expressed as a linear combination of the coefficients for individual orientations. One formulation of this problem is to draw a vector $\vec{a}$ from the origin to the point of interest. State what you mean by the maximization problem. Is not the lambda that is obtained the distance from the origin to the boundary of the hull in the direction of $a$ ? The maximization problem can then be written as

$$
\begin{aligned}
& \lambda=\sum_{i}^{N} \sum_{j}^{P} \frac{a_{i} v_{j} r_{i j}}{\|\vec{a}\|} \\
& \text { Subject to : } \sum v_{j} r_{i j}=\frac{\lambda a_{i}}{\|\vec{a}\|}, \quad \sum_{j}^{P} v_{j}=1, v_{j} \geqslant 0,
\end{aligned}
$$

where $\lambda$ is the value to be maximized, $a_{i}$ is the $i$ th components of the vector $\vec{a}$ within the Fourier space, $r_{i j}$ is the $i$ th component of the vector corresponding to the $j$ th orientation in the set of orientations used to discretize $S O(3) / G, P$ is the number of individual orientations used in the discretization, $v_{j}$ is the volume fraction of the $j$ th point, and $N$ is the number of Fourier dimensions to which the series has been truncated. From linear dependence arguments, it can be shown that no more than $N$ linearly independent points must have non-zero volume fractions for an optimal solution to be obtained. 
Unfortunately, this form of the linear programming problem is not very useful, since the value of the objective appears in the constraints as well as in the objective function.

In order to put the problem into a standard linear programming form, a change of variable is employed and the orthonormal basis of problem is rotated. The volume fractions are replaced by $x_{j}$ defined as

$$
x_{j}=\frac{v_{j}}{\lambda}
$$

and a new basis, $\hat{e}_{i}$, is generated using Gram-Schmidt Orthonormalization where the first basis vector is taken to be $\hat{a}$. The linear programming problem can then be taken to be

$$
\begin{aligned}
& \frac{1}{\lambda}=\sum_{j}^{P} x_{j} \\
& \text { Subject to }: \sum_{j}^{P} A_{i j} x_{j}=\left\{\begin{array}{ll}
1 & \text { if } i=1, \\
0 & \text { otherwise, }
\end{array} \quad x_{j} \geqslant 0,\right.
\end{aligned}
$$

where $A_{i j}$ is the inner product of the $j$ th point with the $i$ th basis vector,

$$
A_{i j}=\left(\hat{e}_{i} \cdot \vec{r}_{j}\right)
$$

The first constraint in Eq. (12) insures that the result (I presume by "result" you mean lambda times vector $a$. If this is correct you might want to replace "result" with the direct expression.) lies completely on the vector $\hat{a}$, where $i=1$. In this form the problem has become one of linear minimization since the objective is still to obtain the largest possible value for $\lambda$. Note that since the zero vector is within the space, a value of $\lambda \geqslant 0$ can always be found. In the form of Eq. (12) the problem can now be solved using general linear programming techniques.

In an attempt to exploit the structure of the problem and thereby increase the efficiency of the linear solutions (Korte and Vygen, 2000), a unique approach has been taken to solve the linear problem. In general, the method will attempt to choose the new basis $\hat{e}_{i}$ in such a way as to get as close to the optimal as possible and then iterate to the optimal solution. To illustrate this approach, consider a problem where four Fourier dimensions are used. Since only four non-zero volume fractions are needed for the solution, the first constraint of Eq. (12) can be written in matrix form as

$$
\left[\begin{array}{cccc}
A_{11} & A_{12} & A_{13} & A_{14} \\
A_{21} & A_{22} & A_{23} & A_{24} \\
A_{31} & A_{32} & A_{33} & A_{34} \\
A_{41} & A_{42} & A_{43} & A_{44}
\end{array}\right]\left[\begin{array}{l}
x_{1} \\
x_{2} \\
x_{3} \\
x_{4}
\end{array}\right]=\left[\begin{array}{l}
1 \\
0 \\
0 \\
0
\end{array}\right] .
$$

The other constraint is simply that the value of $x_{j} \geqslant 0$, and the optimal solution is found when $\sum_{j=1}^{4} x_{j}$ is a minimum. The constraint in Eq. (14) has been formulated 
in terms of the new basis for which only the first basis vector has been defined,

$$
\hat{e}_{1}=\frac{\vec{a}}{\|\vec{a}\|} \text {. }
$$

Let the points $\vec{r}_{1}, \vec{r}_{2}$, and $\vec{r}_{3}$ be used to define the three remaining components of the basis, $\hat{e}_{2}, \hat{e}_{3}$, and $\hat{e}_{4}$, respectively, by successive applications of Gram-Schmidt Orthonormalization. Only the four total vectors $\vec{r}_{1}, \vec{r}_{2}, \vec{r}_{3}$, and $\vec{a}$ are required since four linearly independent vectors always span a four dimensional space. Further, take the point $\vec{r}_{1}$ as the point that has the maximum projection onto $\vec{a}$ of the $P$ points used to discretize $S O(3) / G$. Then the second basis vector is defined as

$$
\hat{e}_{2}=\frac{\vec{r}_{1}-\left(\hat{e}_{1} \cdot \vec{r}_{1}\right) \hat{e}_{1}}{\left\|\vec{r}_{1}-\left(\hat{e}_{1} \cdot \vec{r}_{1}\right) \hat{e}_{1}\right\|} .
$$

Note that if the denominator is zero then $\vec{r}_{1}$ is completely parallel to $\vec{a}$ and the solution to linear optimization problem is given as $\lambda=A_{11}=\vec{r}_{1} \cdot \hat{e}_{1}$. In general this will not be the case, but the vector $\vec{r}_{1}$ resides completely within the first two dimensions of the space and therefore the values of $A_{31}$ and $A_{41}$ are always zero. The third basis vector is then defined as

$$
\hat{e}_{3}=\frac{\vec{r}_{2}-A_{12} \hat{e}_{1}-A_{22} \hat{e}_{2}}{\left\|\vec{r}_{2}-A_{12} \hat{e}_{1}-A_{22} \hat{e}_{2}\right\|} .
$$

Therefore, $\vec{r}_{2}$ resides in a maximum of three dimensions of the space and $A_{42}$ must necessarily be zero. Also note that $A_{32} \geqslant 0$ by definition. The fourth basis vector is then defined as

$$
\hat{e}_{4}=\frac{\vec{r}_{3}-A_{13} \hat{e}_{1}-A_{23} \hat{e}_{2}-A_{33} \hat{e}_{3}}{\left\|\vec{r}_{3}-A_{13} \hat{e}_{1}-A_{23} \hat{e}_{2}-A_{33} \hat{e}_{3}\right\|} .
$$

The constraint in Eq. (14) can now be written as,

$$
\left[\begin{array}{cccc}
A_{11} & A_{12} & A_{13} & A_{14} \\
A_{21} & A_{22} & A_{23} & A_{24} \\
0 & A_{32} & A_{33} & A_{34} \\
0 & 0 & A_{43} & A_{44}
\end{array}\right]\left[\begin{array}{l}
x_{1} \\
x_{2} \\
x_{3} \\
x_{4}
\end{array}\right]=\left[\begin{array}{l}
1 \\
0 \\
0 \\
0
\end{array}\right] .
$$

To this point, only the selection criteria for the first point have been specified. Eq. (19) can be used to determine the criteria for the next three points. Since $A_{21}, A_{32}$, and $A_{43}$ are all defined such that they are positive then $A_{44}, A_{33}$ or $A_{34}$, and $A_{22}, A_{23}$, or $A_{24}$ must be negative in order to satisfy the constraints. Since the next point of interest is $\vec{r}_{2}$, choose an orientation for which $A_{22}$ is negative. More specifically, the linear optimization problem is solved in a sub-dimension. Eq. (19) would appears as

$$
\left[\begin{array}{ll}
A_{11} & A_{12} \\
A_{21} & A_{22}
\end{array}\right]\left[\begin{array}{l}
x_{1} \\
x_{2}
\end{array}\right]=\left[\begin{array}{l}
1 \\
0
\end{array}\right]
$$

if it were only a two-dimensional problem. Immediately it is apparent that in these two dimensions, $A_{22}$ must be negative, since $A_{21}$ was defined to be positive. Solving for $x_{2}$ 
in terms of $x_{1}$ yields

$$
x_{2}=\frac{-A_{21}}{A_{22}} x_{1} .
$$

The value of $x_{1}$ is then given by

$$
x_{1}=\frac{1}{A_{11}-A_{12} A_{21} / A_{22}} .
$$

Therefore $\vec{r}_{2}$ is chosen such that it minimizes

$$
\left(1-\frac{A_{21}}{A_{22}}\right) \frac{A_{22}}{A_{11} A_{22}-A_{12} A_{21}} \text {. }
$$

Once the second point has been selected, the third point can be found, according to Eq. (19), formulated in three dimensions as follows:

$$
\left[\begin{array}{ccc}
A_{11} & A_{12} & A_{13} \\
A_{21} & A_{22} & A_{23} \\
0 & A_{32} & A_{33}
\end{array}\right]\left[\begin{array}{l}
x_{1} \\
x_{2} \\
x_{3}
\end{array}\right]=\left[\begin{array}{l}
1 \\
0 \\
0
\end{array}\right] .
$$

From Eq. (24) the following conditions can be placed on the selection of $\vec{r}_{3}$,

$$
\frac{-A_{32}}{A_{33}}>0, \quad \frac{-A_{21}}{A_{22}-A_{32} A_{23} / A_{33}}>0 .
$$

The constraints are becoming much more complicated as the dimensionality of the space increases. Clearly, for $N$ dimensions, a general form of the constraints must be formulated. The constraints for choosing point $\mathrm{k}$ can be formulated recursively according to

$$
B_{(l+1)}=\frac{-A_{(k-l)(k-l-1)}}{\sum_{m=0}^{l} A_{(k-l)(k-m)} \prod_{n=0}^{l-m} B_{(l-m-n)}}>0 \quad \text { for } l=0 \ldots k-2,
$$

where $B_{0}=1$. In addition to providing the constraints, the solution can be formulated in terms of the $B_{l}$ values. It can be shown that

$$
x_{l+1}=B_{(k-l)} x_{l},
$$

so the value for $x_{1}$ is given by

$$
x_{1}=\frac{1}{A_{11}+\sum_{m=1}^{k} A_{1(m)} \prod_{n=0}^{k-m-1} B_{(k-m-n)}} .
$$

By then repeatedly applying Eq. (27), the value of $\lambda$ can be calculated from Eq. (12). These results are applied for $k=3$, and the point $\vec{r}_{3}$ that maximizes the value of $\lambda$ can be determined by assuming the problem exists in three dimensions only. The process is then repeated for the fourth point by again applying Eqs. (26)-(28) and Eq. (12) with $k=4$. Since the fourth point is the last point, it is optimal, given the choices for the other three points. However, this does not guarantee the optimal solution has been found, since the first three points were not selected with all other points known. 


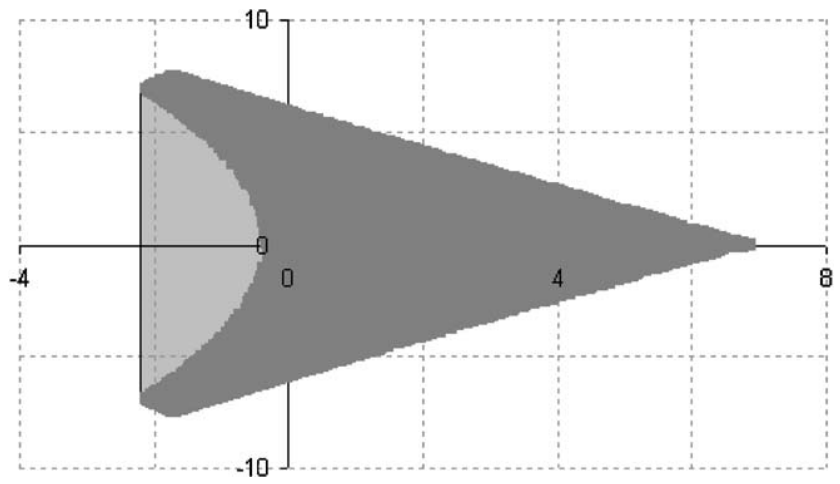

Fig. 2. Two-dimensional slice of the Fourier space showing the location of all possible orientation distribution on the plane $F_{4}^{13}=4$ including a uniquely polycrystalline region denoted by the lighter shade. The $F_{4}^{11}$ and $F_{4}^{12}$ dimensions are shown. Label the axes!

A process of cycling can now be applied to obtain $\mathrm{t}$ the optimal solution. If one now takes $\vec{r}_{2}, \vec{r}_{3}$, and $\vec{r}_{4}$ and rebuilds the basis with them (instead of $\vec{r}_{1}, \vec{r}_{2}$, and $\vec{r}_{3}$ ), one can replace the first point with the optimal, given the choice for the other three. This process is repeated until it has been shown that all points are optimal, with respect to the choices for all other points.

A careful comparison shows that the $B_{l}$ values perform the same role as a pivot in linear algebra when they are applied, as shown in Eqs. (26)-(28). This algorithm uses the $B_{l}$ values in the same way that the simplex algorithm uses pivots (Strayer, 1989), with some small differences in the implementation. The above algorithm must construct the new coordinate frame, which does cost some time in calculations. This effect is countered, to some extent, because the pivoting involves fewer calculations within the new coordinate frame. The differences in the algorithms lie in the way they choose the initial guess points. The above algorithm chooses by solving the problem in a sub-dimension of the linear space. The simplex algorithm uses anticycling rules to govern the selection of the next point. Another subtle difference is in the amount of information that must be stored from iteration to iteration. As $P$ becomes much larger than $N$, the simplex algorithm stores substantially more information (Fig. 2).

The above algorithm was applied to the first three dimensions of the cubicorthorhombic Fourier space. Linear analysis was performed in 2500 directions, using 729,000 distinct orientations to discretize the fundamental zone, corresponding to an accuracy of $1^{\circ}$ in each of the three Euler angles (Bunge, 1993). The result is the convex shape shown as a wire frame in Fig. 3.

The surface of the object is the boundary of the space in which the gradient optimization is taking place. In other words, the boundary of the above shape is the constraint on the gradient optimization, since all microstructures described by points within and on the boundary are real, and all points outside of the hull cannot be matched to real microstructures. 


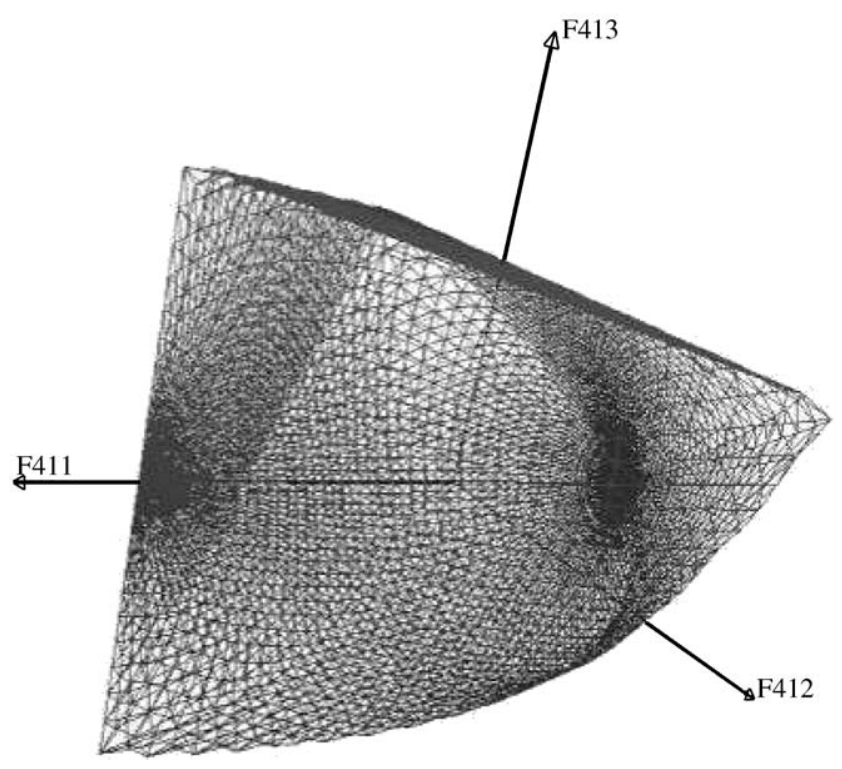

Fig. 3. Wire frame view of microstructure hull in three dimensions.

\section{Leknitskii's anisotropic stress concentration}

As an example of the above gradient optimization method, a thin copper plate with a small hole was analyzed, where the plate was uniaxially loaded along a principal direction of orthotropy. The point of maximum interest is the point where the stress concentration is the highest. Leknitskii (1963) solved for this stress concentration in terms of the components of the compliance tensor. The formula he derived is given in Eq. (29)

$$
\sigma_{t}=\sigma_{a} K=\sigma_{a}\left(1+\sqrt{\frac{4 S_{66}+2 S_{12}}{S_{11}}+2 \sqrt{\frac{S_{22}}{S_{11}}}}\right),
$$

where $\sigma_{t}$ maximum stress around the hole, $\sigma_{a}$ is the applied stress, $K$ is the stress concentration factor, and $S_{i j}$ are particular components of the compliance tensor. The volume average of the local stiffness tensor is its upper bound (Hill, 1952), and this bound will be used to approximate the value for the stress concentration factor. In this case, the stress concentration factor is given by

$$
K=\left(1+\sqrt{\frac{4\left\langle S_{66}\right\rangle+2\left\langle S_{12}\right\rangle}{\left\langle S_{11}\right\rangle}+2 \sqrt{\frac{\left\langle S_{22}\right\rangle}{\left\langle S_{11}\right\rangle}}}\right) .
$$

Since Eq. (30) has a upper bound divided by an upper bound, and since the upper and lower bound come together as the distribution approaches that of a single crystal, the value given by Eq. (30) is predicted to be a reasonable approximation of Eq. (29). The 
Table 1

Summary of minimum and maximum results for stress concentration around a hole in a copper plate

Stress concentrations limits for polycrystalline distributions

Minimum $=2.2215$

Maximum $=3.9477$

Texture coefficients

$F_{4}^{11}=-0.190127$

$F_{4}^{11}=0.086562$

$F_{4}^{12}=-0.853184$

$F_{4}^{12}=0.244637$

$F_{4}^{13}=0.48426$

$F_{4}^{13}=-0.486534$

$J=0.999$

$J=0.304$

volume-averaged components of the stiffness tensor can then be expressed in terms of only the first three Fourier coefficients, without sacrificing any accuracy (Bunge, 1993; Henrie, 2002) in the following manner:

$$
\begin{aligned}
& \left\langle S_{11}\right\rangle=S_{0(11)}^{11}+F_{4}^{11} * S_{4(11)}^{11}+F_{4}^{12} * S_{4(11)}^{12}+F_{4}^{13} * S_{4(11)}^{13}, \\
& \left\langle S_{12}\right\rangle=S_{0(12)}^{11}+F_{4}^{11} * S_{4(12)}^{11}+F_{4}^{12} * S_{4(12)}^{12}+F_{4}^{13} * S_{4(12)}^{13}, \\
& \left\langle S_{22}\right\rangle=S_{0(22)}^{11}+F_{4}^{11} * S_{4(22)}^{11}+F_{4}^{12} * S_{4(22)}^{12}+F_{4}^{13} * S_{4(22)}^{13}, \\
& \left\langle S_{66}\right\rangle=S_{0(66)}^{11}+F_{4}^{11} * S_{4(66)}^{11}+F_{4}^{12} * S_{4(66)}^{12}+F_{4}^{13} * S_{4(66)}^{13} .
\end{aligned}
$$

With this model developed, the gradient-based optimization that utilizes the above linear programming method was applied. Since only the first three Fourier coefficient are important in this problem, $N=3$. Local minima and maxima did presented a significant challenge for the model, so multiple initial starting points were utilized. The minimum value for $K$ was found to be 2.215 .

Since the goal of MSD is to obtain a solution that is produced by common processing routes, the texture severity index factor (Sturcken and Croach, 1963) was introduced to give a rough estimate of the cost to produce the specified microstructure. The texture severity index, $J$, is calculated by

$$
J=\sum_{l=4}^{\infty} \sum_{m=1}^{M(l)} \sum_{n=1}^{N(l)} F_{l}^{m n} F_{l}^{m n} .
$$

The value of $J$ will range from 0 , for a completely random microstructure, to $\infty$, for a perfect single-crystal microstructure. The minimum and maximum values of $\mathrm{K}$ are shown in Table 1, along with the Fourier coefficients of the solution and the texture severity index value.

Notice that the values for the texture severity index do not approach what they would for a single-crystal microstructure. This is due to the truncation of the Fourier series, which forces solutions that are relatively smooth distributions. Truncation of the Fourier series will always result in a microstructure with a smoother distribution, which is often easier to produce by conventional processing. The local minimum and local maximum, which were obtained when the starting location for the optimization was a completely random microstructure, also provided interesting solutions to the microstructure design 
Table 2

Local minimum and maximum found from initially random texture showing the stress concentration around a hole in a copper plate

Stress concentrations limits for polycrystalline distributions

Local minimum $=2.332$

Local maximum $=3.9474$

Texture coefficients

$F_{4}^{11}=-0.123692$

$F_{4}^{12}=-0.399752$

$F_{4}^{13}=0.495438$

$F_{4}^{11}=0.02865$

$F_{4}^{12}=0.25815$

$J=0.421$

$F_{4}^{13}=-0.468842$

$J=0.287$

problem. The initial values of the Fourier coefficient were all zero for the solutions that are shown in Table 2.

A large difference is found between the local minimum and the global minimum in terms of the texture severity index, although the increase in stress concentration is less than 5\%. The local and global maximums were closer together, and yet some difference in the texture severity index was obtained with negligible effect on the stress concentration. The optimization could therefore include the texture severity index value, as long as its importance relative to the stress concentration value is known.

\section{Taylor yield theory}

When one adjusts the value of the stress concentration factor, it is also important to know if the derived orientation distribution also lies among the weakest yield strengths. In this case, any gains in stress concentration factor could be undermined by reductions in yield strength. In that which follows, Taylor yield theory was used to assure that the solution has the best possible yield strength to stress concentration ratio, which would allow the largest stress to be applied to the plate. The following development follows the work of Bunge (1979) to develop the Taylor factor within the Fourier space of the cubic-orthorhombic generalized spherical harmonic functions. In full-constraints Taylor theory, every grain in the sample undergoes the same deformation. For the copper plate with cubic-orthorhombic symmetry, loaded in directions coincident with the principal axes of the anisotropy, a strain in the following form can be assumed:

$$
\varepsilon_{i j}=\left[\begin{array}{ccc}
1 & 0 & 0 \\
0 & -q & 0 \\
0 & 0 & -(1-q)
\end{array}\right]
$$


Table 3

Summary of results for a copper plate with a circular hole analyzed according to Taylor factor and stress concentration factor

Maximum Taylor factor to stress concentration ratio

Maximum $M / K$ ratio $=1.228$

Taylor factor $=3.538 \quad$ Stress concentration $=2.881$

Texture coefficients

$F_{4}^{11}=-0.462041$

$$
\begin{aligned}
& F_{6}^{14}=0.547706 \\
& F_{8}^{11}=0.167714 \\
& F_{8}^{12}=-0.356061 \\
& F_{8}^{13}=-0.211022 \\
& F_{8}^{14}=-0.605061 \\
& F_{8}^{15}=0.041599
\end{aligned}
$$$$
F_{10}^{11}=0.333715
$$

$F_{4}^{12}=0.258893$

$F_{10}^{12}=-0.27499$

$F_{4}^{13}=0.172904$

$F_{10}^{13}=-0.138081$

$F_{6}^{11}=-0.485852$

$F_{10}^{14}=0.283281$

$F_{6}^{12}=-0.123046$

$F_{10}^{15}=0.176355$

$F_{6}^{13}=-0.221207$

$F_{10}^{16}=0.186187$

$J=1.830$

If the value of $q$ is known then the Taylor factor can be expressed as a Fourier series

$$
M(q)=\sum_{l=0}^{\infty} \sum_{m=1}^{M(l)} \sum_{n=1}^{N(l)} \frac{F_{l}^{m n} m_{l}^{m n}(q)}{(2 l+1)} .
$$

For the copper plate, the stress conditions imposed by the Leknitskii's solution must be satisfied and therefore the value of $q$ must be determined such that it satisfies those stress conditions. This can be accomplished by finding the minimum of $M(q)$ as $q$ goes from 0 to 1 for the strain given in Eq. (33) and the uniaxial stress state. Bunge took the approach of expressing the $q$ dependence as a power series yielding the following equation

$$
M(q)=\sum_{l=0}^{\infty} \sum_{m=1}^{M(l)} \sum_{n=1}^{N(l)} \sum_{\zeta=0}^{R} \frac{F_{l}^{m n} m_{l \zeta}^{m n} q^{\zeta}}{(2 l+1)} .
$$

Eq. (35) can be easily differentiated in order to determine the value of $q$ that minimizes the Taylor factor. Clearly the value of $q$ is a function of texture, and therefore Eq. (34) is non-linear with respect to the Fourier coefficients, since it is dependent on $q$ and the texture coefficients. Bunge showed that by taking the order $l=10$ and $R=6$, the Taylor factor becomes fairly accurate (1979). This yields a Fourier space of dimension $N=18$ for the cubic-orthorhombic case. During this study the accuracy of Bunge's results was verified, and the maximum ratio of Taylor factor to stress concentration was sought. For the minimum ratio the order of the series would need to be increased to get accurate results. As a reference value, the minimum ratio of 0.6 , obtained by Henrie et al. (2002) by simulated annealing, was used. The analysis was conducted to determine the orientation distribution that would increase the ratio of the Taylor factor to the stress concentration factor the most. The global results, obtained from starting the algorithm at a variety of initial points, are shown in Table 3, and the 
Table 4

Summary of the local maximum ratio results for a copper plate with a circular hole analyzed according to Taylor factor and stress concentration factor from an initial random microstructure

Local maximum Taylor factor to stress concentration ratio

Local maximum $M / K$ ratio $=1.166$

Taylor factor $=3.295 \quad$ Stress concentration $=2.825$

Texture coefficients

$F_{4}^{11}=-0.232104$

$F_{6}^{14}=-0.094158$

$F_{10}^{11}=0.010208$

$F_{4}^{12}=-0.007683$

$F_{8}^{11}=0.077224$

$F_{4}^{13}=0.133426$

$F_{8}^{12}=-0.084573$

$F_{10}^{12}=-0.020275$

$F_{6}^{11}=-0.231691$

$F_{8}^{13}=0.075185$

$F_{10}^{13}=0.007077$

$F_{6}^{12}=-0.070378$

$F_{8}^{14}=-0.071094$

$F_{10}^{14}=-0.000459$

$F_{6}^{13}=0.047469$

$F_{8}^{15}=0.103469$

$F_{10}^{15}=0.013528$

$J=0.178$

local results obtained from starting at only an initially random position are shown in Table 4.

Several interesting points can be made about these results. First, the maximum ratio is only about $20 \%$ larger than it would be for a completely random distribution, and yet more than $100 \%$ larger than the minimum ratio. It is also interesting to note that neither the stress concentration factor nor the Taylor factors are at their lower or upper limits in the above results (the limits for $K$ are given in Table 1; the maximum $M$ is approx. 3.67). The interaction between the two properties is complex, and the solution could not have been predicted if the properties had been considered independently.

As with the stress concentration factor alone, the local maximum Taylor factor to stress concentration ratio has nearly the same performance as the global maximum, but with a significantly lower texture severity index value. The local maximum has a decrease in performance of only $5 \%$ with a texture severity index value more than 10 times smaller than that of the global maximum. The pole figures for the textures in Tables 3 and 4 are shown in Figs. 4 and 5, respectively.

The maximum values for both graphs are relatively low, due to truncation of the Fourier series to order $l=10$. The larger of these two values occurs in the graph of the texture with the lower texture severity factor. Although the center plot in Fig. 5 does show a smoother texture, the left plot has a significant peak value. Fig. 4, in contrast, has significant peaks in all three plots. Clearly, the texture severity factor does not contain all of the information one would need in selecting the microstructure that is easiest to produce.

As stated in the introduction, these results reflect only what is theoretically possible and not necessarily what we can now obtain. Eventually a catalog of data could be collected from specific material processing methods and use that to limit the algorithm optimization paths and therefore constrain the solutions to feasible microstructures. 

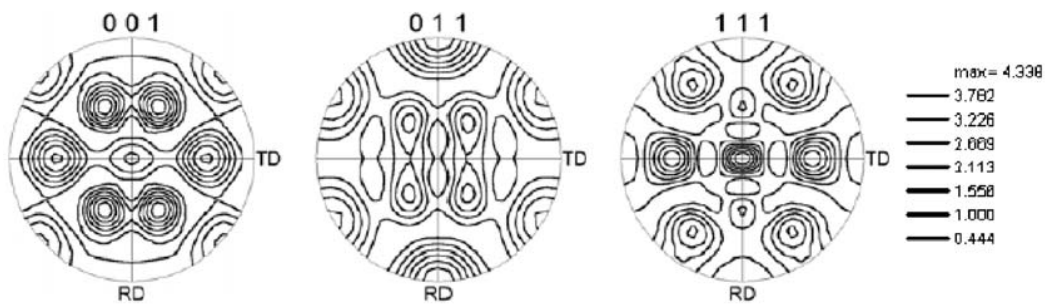

Fig. 4. Pole figures with $5^{\circ}$ Gaussian smoothing for the texture given in Table 3, corresponding to the global optimum for the Taylor factor to stress concentration ratio.

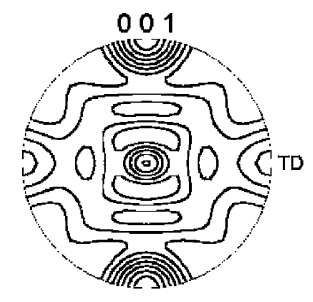

RD

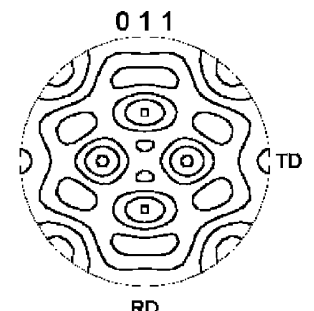

RD

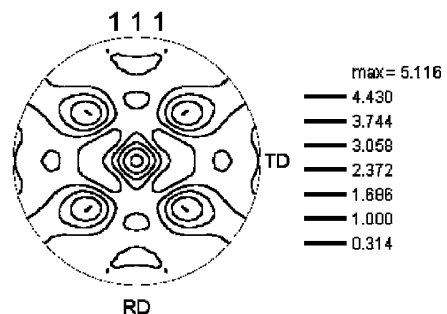

RD

Fig. 5. Pole figures with five degree Gaussian smoothing for the texture given in Table 4, corresponding to a local optimum for the Taylor factor to stress concentration ratio.

Some of this data is currently available but to fully utilize this method, significantly more processing data needs to be collected.

\section{Summary}

A formulation for the linear programming problem of defining the convex boundary of the Fourier coefficients for real orientation distribution functions is presented, along with a method based on Gram-Schmidt Orthonormalization for solving it. Determining this boundary allowed gradient-based optimization methods to be used within the Fourier space to determine optimal orientation distribution functions for given sets of constraints or property requirements. The above method was applied to a thin copper plate with a small circular hole possessing orthotropic symmetry. Both the Taylor factor and the stress concentration factor around the hole were analyzed, and the orientation distributions for extremal points determined. Local minimum and maximum values were also found that presented microstructures alternate solutions that could be easier to produce. Through the methods presented, a designer is able to take advantage of the natural anisotropy of the material, without being forced to use expensive single-crystal components by defining constraints for the specific application and available processing methods. 
Current research involves extending this method to higher order correlations statistics including two-point statistics. For the case of two-point statistics, the methods can be extended directly for a single vector direction and length. The correlations between vectors of different lengths and directions require additional and non-linear constraints in order to obtain necessary and sufficient condition for the existence of a microstructure with the predicted statistics.

\section{Acknowledgements}

The support of this research by the Army Research Office is gratefully acknowledged. M. Lyon is supported by a National Science Foundation Graduate Research Fellowship.

\section{References}

Adams, B.L., Henrie, A., Henrie, B., Lyon, M., Kalidindi, S.R., Garmestani, H., 2001. Microstructure-sensitive design of a compliant beam. J. Mech. Phys. Solids 49, 1639-1663.

Adams, B.L., Lyon, M., Henrie, B., 2004. Microstructures by design: linear problems in elastic-plastic design. Int. J. Plasticity 20, 1577-1602.

Brocker, T., tom-Dieck, T., 1985. Representations of Compact Lie Groups. Springer, New York.

Bunge, H.J., 1965. Zur Darstellung allgemeiner Texturen. Z. Metallkd. 56, 872-874.

Bunge, H.J., 1979. Textur and Anisotropie. Z. Metallkd. 70, 411-417.

Bunge, H.J., 1993. Texture Analysis in Material Science. Cuvillier Verlag, Goettingen.

Henrie, B.L., 2002. Elasticity in microstructure sensitive design through the use of Hill bounds. Thesis, Brigham Young University, Provo, UT.

Henrie, B.L., Lyon, M., Adams, B.L., 2002. Microstructure engineering in design. Ninth AIAA/ISSMO Symposium on Multidisciplinary Analysis and Optimization. AIAA Paper Number 2002-5567.

Hill, R., 1952. The elastic behavior of a crystalline aggregate. Proc. Phys. Soc. London A 65, 349-354.

Kalidindi, S.R., Houskamp, J.R., Lyon, M., Adams, B.L., 2002. Microstructure sensitive design of an orthotropic plate subjected to tensile load. Int. J. Plasticity 20, 1561-1575.

Keener, J.P., 2000. Principles of Applied Mathematics. Perseus Books, Cambridge, MA.

Korte, B., Vygen, J., 2000. Combinatorial Optimization. Springer, Berlin.

Leknitskii, S.G., 1963. Theory of Elasticity of an Anisotropic Elastic Body. Holden-Day, San Francisco.

Rardin, R., 1998. Optimization in Operations Research. Prentice-Hall, New Jersey.

Roe, R.J., 1965. Description of crystallite orientation in polycrystalline materials, III. General solution to pole figure inversion. J. Appl. Phys. 36, 2024-2031.

Strayer, J.K., 1989. Linear Programming and its Applications. Springer, New York.

Sturcken, E.F., Croach, J.W., 1963. Predicting physical properties in oriented materials. Trans. Met. Soc. AIME 227, 934-940. 